Colloque C3, suppl, au Journal de Physique III, Vol. 1, octobre 1991

\title{
HIGH STRAIN RATE TESTING OF MATERIALS - A FULLY VALIDATED TEST CALIBRATION BY A HYBRID NUMERICAL/EXPERIMENTAL TECHNIQUE
}

\author{
D.J. RUIZ(1), J. HARDING and C. RUIZ
}

Department of Engineering Science, University of Oxford, Parks Road, GB-Oxford ox1 3PJ, Great-Britain

\begin{abstract}
Résumé: Certaines conclusions d'une ćtude complète expćrimentale ct numérique sur un test de cisaillement à doublc entaille (DNS) pour la détermination du comportement des matériaux à très grande vitesse de déformation sont décrites. La photographic ultrarapide, la photoélasticjté dynamique et l'analyse numérique par la méthode des éléments finis ont ćté utilisées. Le test DNS est calibré et on en tirc le conclusions sur l'orientation future des essais de matériaux à grande vitesse.

Abstract - Some of the conclusions of an extensive experimental and numerical study of the double-notch shear (DNS) test for the determination of material behaviour at very high rates of strain are described. Ultra-high speed photography, dynamic photoelasticity and the finite element method of numerical analysis are used. The DNS test is calibrated and conclusions are drawn on the direction of future high strain rate material testing.
\end{abstract}

\section{Introduction}

In an increasing number of engineering problems structures are subjected to extreme loading conditions. Of these conditions impact is probably the most common. Accurate and reliable quantification of material behaviour at impact rates of strain, i.e. $10^{3} / \mathrm{s}$ and above, is therefore necessary in the interests of efficient design.

The DNS test was devised more than 20 years ago [1] to facilitate the collection of data on the mechanical behaviour of materials in pure shear at strain rates of the order of $10^{4} / \mathrm{s}$. The test is based on the split Hopkinson pressure bar (SHPB) technique. Specimen geometry, especially in high strain rate tests, is sometimes thought to play a major part in causing the rate sensitive response observed in material tests. It is therefore highly desirable to perform a comprehensive study of the DNS test using advanced experimental methods in conjunction with numerical analysis. Only over the last decade or so have numerical analysis capabilities developed sufficiently to cope with the highly non-linear problems posed by impact.

Using this hybrid method of analysis the DNS test has been calibrated so that the test data obtained can be correlated with that from tension and compression tests. Results are presented for annealed OFHC copper over a range of strain rates from $10^{-3}$ to $10^{+4} / \mathrm{s}$ and general conclusions are drawn on future material testing at high rates of strain. The work is discussed in greater detail in [2].

(1) Now at Ove Arup and Partners, 13 Fiztroy Street, GB-London

WIP 6BQ, Great-Britain. 


\section{The Double-Notch Shear (DNS) Test}

The SHPB technique uses elementary longitudinal elastic wave theory to derive the force and the velocity histories at the interfaces between the specimen and the input and output loading bars. By assuming a state of

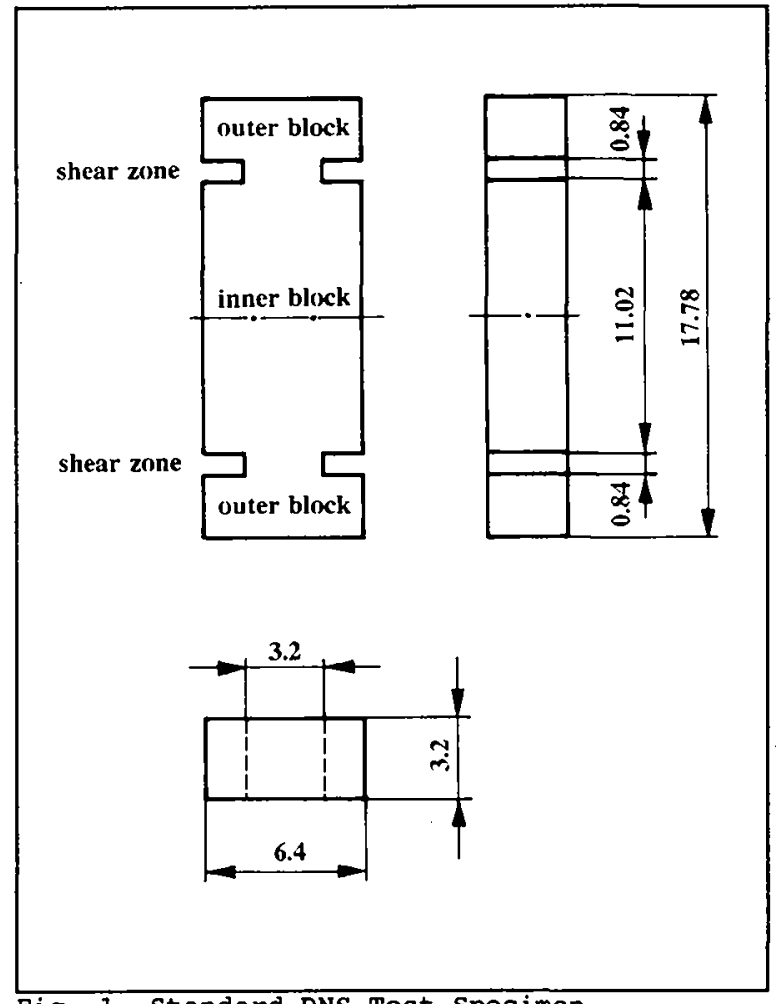

Fig. 1 standard DNS Test specimen

$$
\sigma=\sqrt{ } 3 \tau_{n}
$$

and quasi-static equilibrium and the concentration of the deformation to a geometrically defined zone within the specimen the force and velocity histories may be converted to stress and strain rate histories. Fig. 1 shows the standard DNS specimen.

This is sandwiched between an input bar and an output tube. All the strain is assumed to be confined to the shear zone, giving a shear zone width of $0.84 \mathrm{~mm}$. The external force is applied over two equal areas of $(3.2 \mathrm{~mm})^{2}$. Hence the nominal shear stress and the nominal shear strain, respectively, are given by

and

$$
\begin{aligned}
& \gamma_{n}=(6.4-\Delta) / 0.84 \\
& \tau_{n}=F / 2(3.2)^{2}
\end{aligned}
$$

where $F$ is the applied load and $\Delta$ is the separation between the loading bars. To correlate the shear data with that from either tension or compression tests the von Mises criterion is assumed so that the effective stress and strain are given by

$$
\epsilon=\gamma_{n} / \sqrt{ } 3
$$

\section{Material Tests}

Tests have been performed in tension, compression and double-notch shear on OFHC copper, annealed at $800^{\circ} \mathrm{C}$, at strain rates from $10^{-3}$ to $10^{44} / \mathrm{s}[2]$. Effective stress-effective strain curves illustrating the mean response observed in these various tests at five different strain rates are compared in fig. 2. The low and medium rate tensile tests show the usual increase in flow stress with strain rate and correlate well with the slightly higher rate compression tests [3]. Considerable experimental scatter was obtained at low plastic strains in the highest rate tensile tests. Only at strains above $\sim 20 \%$ do these results show correlation with the other uniaxial tests. While the DNS results, at a nominal strain rate of about $10^{4} / \mathrm{s}$, showed very little experimental scatter, the corresponding effective flow stresses were well below those for thee uniaxial tests. Also initial yield in the DNS tests appeared to be at a nominal shear strain of between 5 and $10 \%$. It was observed that at higher overall displacements plastic deformation in the DNS specimen was not confined to the shear zone but was also apparent at the interfaces between the specimen and the output tube and was associated with a significant rotation of the specimen end blocks so that the deformation process was no longer one of pure shear. 
In the light of these observations it is clear that a full evaluation of the DNS test is required so that the validity of the data obtained can be assessed. The aim of the present study, therefore, is to monitor as fully as possible the structural response of the DNS specimen and to relate this to its high rate material properties.

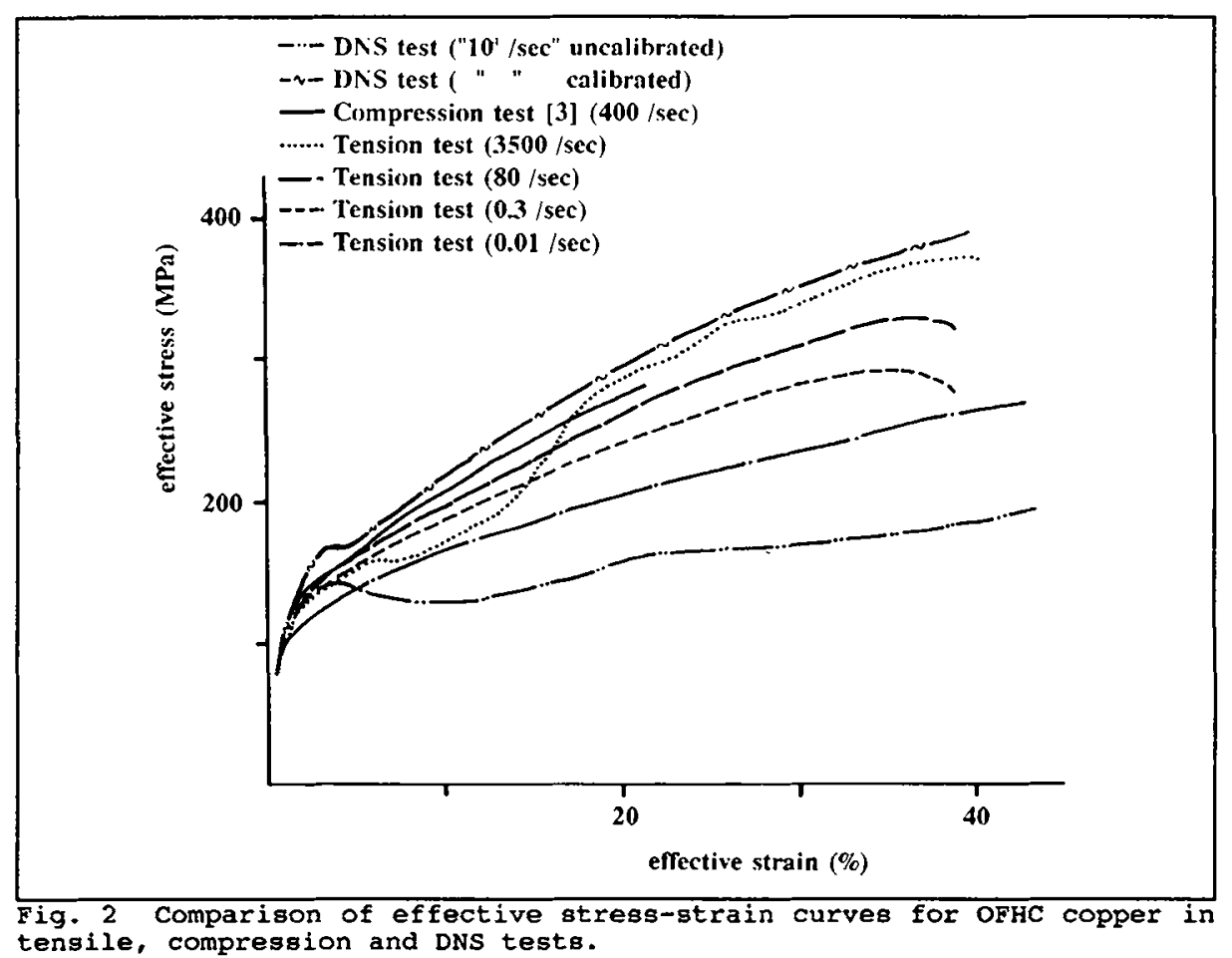

\section{Evaluation of the DNS Test}

Experimental Work Using dynamic photoelasticity and ultra-high speed photography full field records of the elastic and elasto-plastic response of the specimen have been obtained in addition to the usual Hopkinson bar strain gauge data. The photoelastic experiment was used to obtain elastic stress contours in a model DNS specimen [4] and the high-speed photography to record the displacement field for the specimen during the test. These data have been used firstly as a check on the SHPB analysis and secondly to establish the validity of the numerical analysis described below. Fuller details of this work are given elsewhere $[2,4,5]$.

The photoelastic experiment demonstrated that there are no significant wave propagation effects within the specimen so that quasi-static equilibrium is quickly established and the SHPB analysis is basically valid. The high-speed photographs confirmed that, in this particularly ductile material, significant plastic deformation occurs at the output faces of the specimens in addition to that in the shear zone. There is also both a sliding movement and a separation between the specimen output face and the output tube as the end blocks rotate. Nevertheless, despite these problems, a major advantage of the DNS test is the planar specimen geometry which lends itself to the use of advanced full-field monitoring techniques.

Numerical Analysis The DNS test has been analysed in three stages using the general purpose Lagrangian finite element code ABAQUS. In the first 
stage the results of the photoelastic experiments were modelled using large displacement theory with linear boundary conditions and assuming a simple linear elastic material model based on the properties of the epoxy resin used to make the photoelastic specimen. In stage two a rate-independent elasto-plastic material model replaced the material model of stage one and non-linear boundary conditions were used, to allow

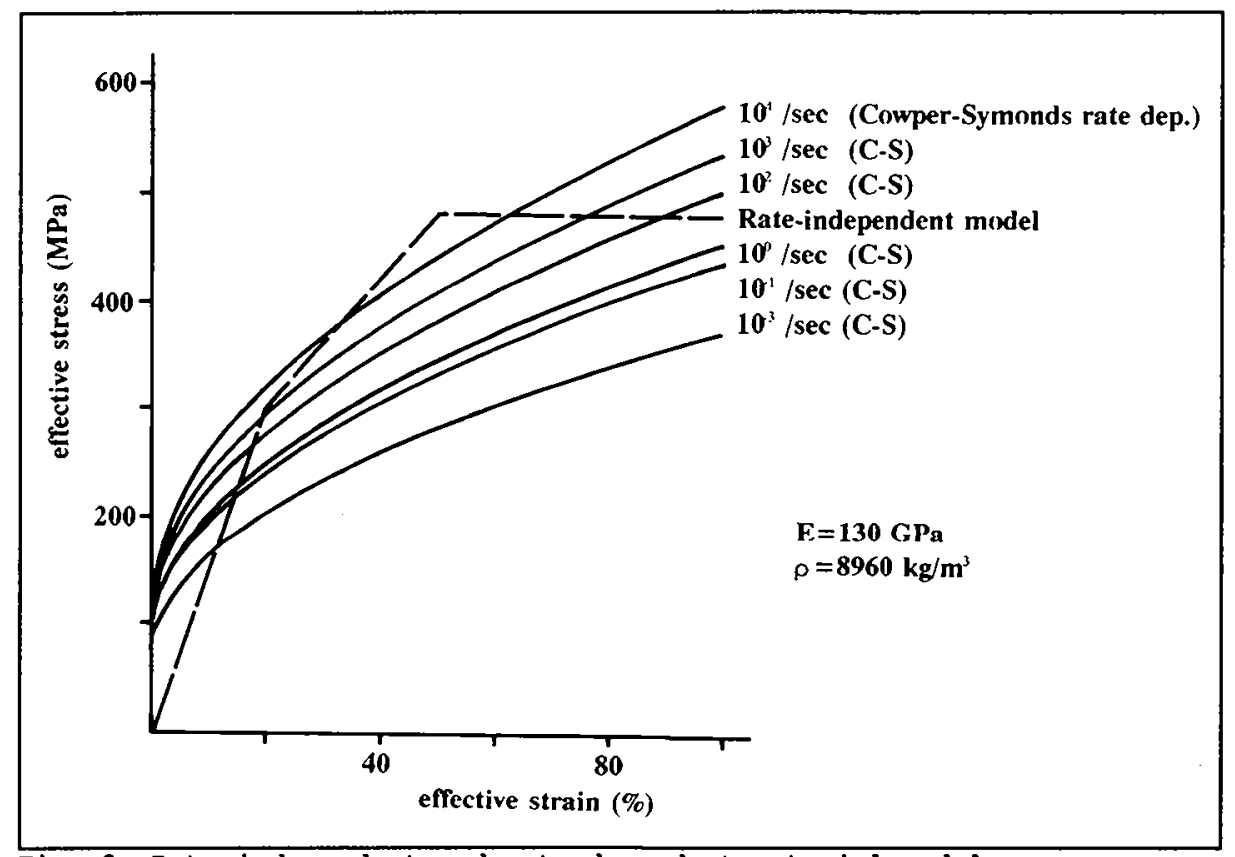

Fig. 3 Rate-independent and rate-dependent material models

for the loss of contact at high deformations. Comparison was made with the high-speed photographic data and with material tests. Stage three repeated stage two but used the Cowper-symonds rate-dependent material model. Only the specimen was modelled. A simplified velocity history, related to that derived from the SHPB analysis for a typical DNS test, was applied uniformly to the nodes on the specimen input face and the output face was assumed to be in contact with a rigid surface. The rate-independent material model, previously used with some success by Baker et al. [6], is compared in fig. 3 with stress-strain curves at different strain rates derived from the Cowper-Symonds rate-dependent model using empirical constants obtained from the uniaxial material tests of fig. 2 .

\section{Results}

Calibration of the DNS test By comparing the mean stress along the notch centre line, $\sigma_{\mathrm{mm}}$ as derived from the FEM analyses, with the nominal shear stress, $\tau_{n}$, derived from the SHPB analysis, a stress calibration factor, $\mathrm{K}_{o}$, can be derived. Similarly a strain calibration factor, $\mathrm{K}_{\epsilon}$, is obtained from a comparison of the nominal shear strain with the mean shear strain along the centre line of the notch. Although these factors vary during the course of the test, for times $<~ 10 \mu s$ they are relatively constant. When used to correct the original DNS data in fig. 2 they give an effective stress-strain curve which correlates much more closely with those obtained from the uniaxial stress tests. 
Modelling the displaced shape of the DNS specimen A comparison between the displaced shape of the DNS specimen as recorded by the high-speed camera with that predicted by the rate-dependent finite element analysis is given in fig. 4. Very good agreement is obtained.

Predicting the reaction force history similarly a comparison is made in fig. 5 of the experimentally determined force history on the output face

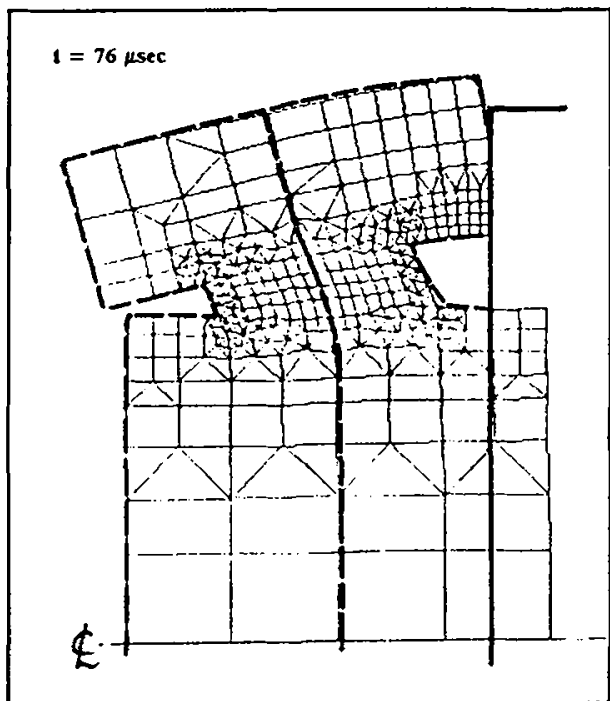

Figure Deformed DNS specimen: comparison of high-speed photograph with deformed finite element mesh (outline of high-speed photograph --$---)$

of the specimen with those predicted by both the rate independent and the rate dependent finite element analyses. The rate dependent model clearly gives a much closer agreement with experiment than does the rate independent one, a1though some differences are apparent in the very early stages of the test.

\section{Discussion}

Although it is clear from the high speed photographs that only over a very limited range of strains does the DNS specimen deform in pure shear and that as the test proceeds a significant proportion of the overall displacement applied across the input and output faces of the specimen appears as plastic deformation outside the shear zone, nevertheless by the use of the calibration factors $k_{o}$ and $K_{\epsilon}$ a reasonable correlation may be obtained between the effective stressstrain curves derived from the DNS test

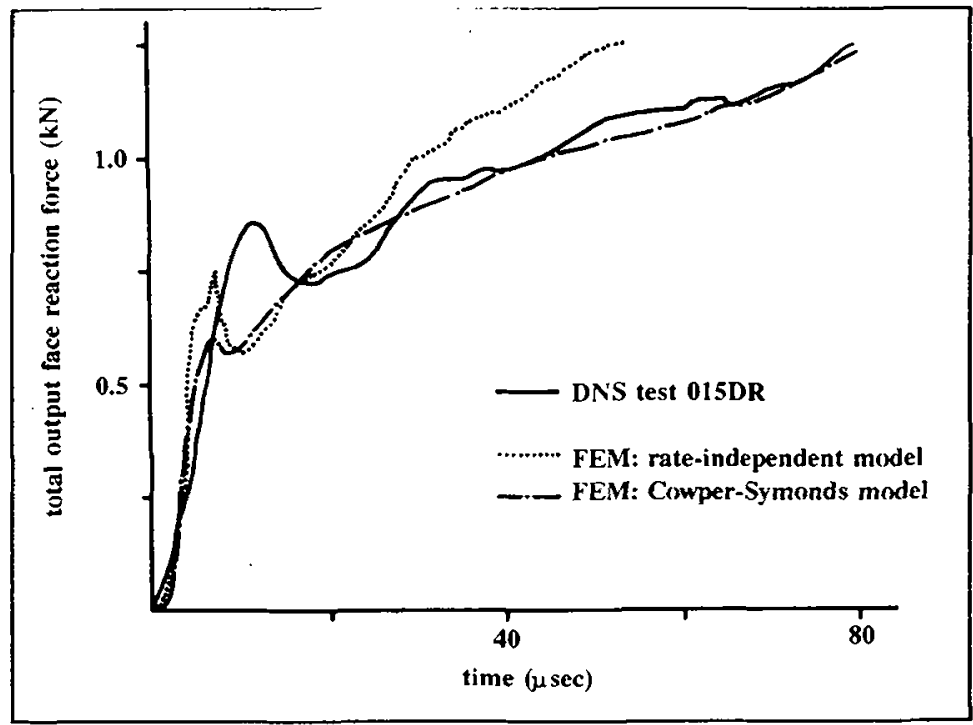

Fig. 5 Comparison of reaction force histories and those obtained using standard uniaxial tension or compression tests.

However, since the calibration factors are themselves derived using the finite element analysis and so depend on the choice of material model, an independent check is required on the validity of the $F E$ analysis and, in particular, on the choice of material model. Such checks may be made in terms of the ability of the analysis either to model the deformed shape of the dynamically loaded testpiece, the approach that is more usually followed, or to predict the loads required to produce this deformation. 
It is apparent from fig. 4 that the finite element analysis using the rate-dependent material model predicts very closely the deformed shape of the DNS specimen. However, when the displacement boundary conditions are prescribed the predicted displacement field is unlikely to be sensitive to the precise form of the material model used. This was shown to be the case in the present investigation when the predicted transverse centre-line displacement of the DNS specimen was found to be in equally good agreement with the experimental measurements from the high-speed photographs whether the rate-dependent or the rate-independent material model was used [2].

A more sensitive check, however, is in terms of the forces required to produce the dynamic deformation. Since in the finite element analysis the stress field is deduced from the nodal displacements, using a stiffness matrix which incorporates the chosen material model, the forces predicted by the finite element analysis are likely to be strongly dependent on the model chosen. Using the SHPB technique it is possible to make an accurate experimental measurement of the force history on the output faces of the DNS specimen. This may then be compared with the predictions of the finite element analysis. Such a comparison is given in fig. 5 from which it is clear that the rate-dependent material model, unlike the rate-independent model, predicts fairly closely the observed force history at times $>30 \mu \mathrm{secs}$. Neither model predicts the experimentally determined force history at times < $20 \mu \mathrm{secs}$. This may be because the assumption of quasi-static equilibrium within the specimen is likely to be most in error in the very early stages of the test. It is also likely that an ability to model the discontinuous yielding associated with an apparent "yield drop" is an even more sensitive test of the choice of material model. Since it is clear that the rate-dependent material model used here has significant limitations [7], the zerilliAmstrong model, which has a more fundamental scientific basis [8], has been incorporated into ABAQUS. In the present application, however, it is much slower to run than the cowper-symonds model already built-in to ABAQUS so, as yet, it has not been possible to use it.

\section{Conclusions}

By a combination of experimental (high-speed photographic) and numerical (finite element) techniques a detailed study has been made of the DNS test and calibration factors have been determined for tests on annealed OFHC copper. Although it is clear that the DNS test does not measure high rate material properties in pure shear it has been shown to provide a sensitive method for comparing the validity of different models of material behaviour when used in finite element codes.

\section{References}

1. J. D. Campbell and W. G. Ferguson, Phil. Mag., 21, 1970,.63-82.

2. D. J. Ruiz, D. Phil. Thesis, Oxford University, 1990.

3. N. A. Safford, Cavendish Laboratory, University of Cambridge (private communication).

4. D. J. Ruiz, J. Harding and C. Ruiz, in Proc. $1^{\text {st }}$ Int. Conf. on Structures under shock and Impact, SUSI1, ed. P. S. Bulson, (Elsevier, London and New York) 1989, 145-154.

5. D. J. Ruiz, J. Harding, J. Noble and G. Hillsdon, in Proc. $19^{\text {th }}$ Int. Congress on High Speed Photography and Photonics, Cambridge, September 1990 (SPIE, Soc. of Photooptical Instrumentation Engineers).

6. S. Baker, R. Bateman and P. Church, MOD RARDE Memorandum 5/88, 1988.

7. J. Harding, in Proc. $4^{\text {th }}$. Oxford Int. Conf. on Mech. Props. of Materials at High Rates of Strain, Inst. of Physics Conf. Series No. 102, (Inst. of Physics, London and Bristol), 1989, 189-203.

8. F. J. Zerilli and R. W. Armstrong, J. Appl. Phys., 61, No. 5, 1987, 1816-1825. 